\title{
Therapeutic effects of a liquid bandage prepared with cellulose powders from Styela clava tunics and Broussonetia kazinoki bark: Healing of surgical wounds on the skin of Sprague Dawley rats
}

\author{
JIN JU PARK ${ }^{1}$, JI EUN KIM ${ }^{1}$, WOO BIN YUN ${ }^{1}$, MI RIM LEE ${ }^{1}$, JUN YOUNG CHOI ${ }^{1}$, \\ BO RAM SONG ${ }^{1}$, HONG JOO SON ${ }^{1}$, YONG LIM ${ }^{2}$, HYUN-GU KANG ${ }^{3}$, BEUM SOO AN ${ }^{1}$, \\ SEUNG YUN YANG ${ }^{1}$, SUNG BAEK SEO $^{1}$ and DAE YOUN HWANG ${ }^{1}$
}

\author{
${ }^{1}$ Department of Biomaterials Science, College of Natural Resources and Life Science/Life and Industry Convergence \\ Research Institute, Pusan National University, Miryang, Gyeongsangnam-do 50463; ${ }^{2}$ Department of Clinical \\ Laboratory Science, College of Nursing and Healthcare Science, Dong-Eui University, Busan 47340; \\ ${ }^{3}$ Veterinary Medical Center and College of Veterinary Medicine, Chungbuk National University, \\ Cheongju, North Chungcheong 28644, Republic of Korea
}

Received January 5, 2018; Accepted October 19, 2018

DOI: $10.3892 / \mathrm{mmr} .2018 .9668$

\begin{abstract}
Cellulose in different forms has extensively been applied in biomedical treatments, including scaffolding, tissue engineering and tissue formation. To evaluate the therapeutic effects of a liquid bandage (LB) prepared with cellulose powders from Styela clava tunics (SCT) and Broussonetia kazinoki bark (BSLB) for healing cutaneous wounds, the remedial effects of a low concentration (LoBSLB) and a high concentration (HiBSLB) of BSLB on skin regeneration and toxicity in Sprague Dawley rats. Results indicated that the total area of skin involved in the surgical wound was lower in the BSLB-treated group compared with the Vehicle-treated group at days 4-12, although some variations were observed in the HiBSLB-treated group. In addition, the BSLB-treated group showed significantly enhanced width of the re-epithelialization region and epidermal thickness when compared with the Vehicle-treated group. Furthermore, significant stimulation in the expression level of collagen-1 and the signaling pathway of VEGF after topical application of BSLB was indicated. No liver or kidney toxicities were detected for either doses of BSLB. Overall, the results of the present study suggest that BSLB accelerates the process of wound healing in surgical skin wounds of Sprague Dawley rats through stimulation of re-epithelialization and connective tissue formation, without any accompanying significant toxicity.
\end{abstract}

Correspondence to: Professor Dae Youn Hwang, Department of Biomaterials Science, College of Natural Resources and Life Science/Life and Industry Convergence Research Institute, Pusan National University, 50 Cheonghak-ri, Samnangjin-eup, Miryang, Gyeongsangnam-do 50463, Republic of Korea

E-mail: dyhwang@pusan.ac.kr

Key words: Styela clava tunics, Broussonetia kazinoki bark, cellulose, surgical wound, toxicity

\section{Introduction}

Cellulose, composed of repeating glucose units connected by $\beta$ - $(1,4)$-glycosidic bonds, is one of the most abundant among the many natural polymers and is one of the major organic compounds found in plant cell walls $(1,2)$. Due to its white fiber-like structure, no odor and a density of $\sim 1.5$, cellulose and its derivatives are commonly used as drug coating materials, blood coagulants, artificial kidney membranes, antitumor drugs, blood-compatible materials, and supports for immobilized enzymes (3-7). Furthermore, no special treatments are required for eliminating risk factors such as immune response and viral risk, which are observed after chitosan treatment (8-11). Cellulose membranes have especially been applied in medical devices such as dialysis machines and biosensors, while bioadhesive cellulose gels have an application in bone tissue engineering and connective tissue formation (12). However, very little is known about the possibility of LB prepared with cellulose powders originating from the different natural sources such as marine animals and plants.

LB is a chemical mixture which forms a thin polymeric layer on the skin; this characteristic has found a use as a topical skin treatment for minor cuts and sores (13). In humans, it stimulates the skin wound repair by retaining an adequate moisture balance and protecting the wound from infections $(10,14)$. Extensive preparations of liquid bandage (LB) using various other polymers have been used, including polyvinylpyrrolidone, ethyl cellulose, pyroxylin/nitrocellulose, poly (methylacrylate-isobutene-monoisopropylmaleate), and acrylate or siloxane polymers (13). 2-octyl-cyanoacrylate is the most common polymer for preparing LB applied to cornea, sclera, eyelid skin grafts, and mucous membrane grafts during socket reconstruction, and for temporary treatment of myopathic blepharoptosis after botulinum toxin injection (15-19). Studies involving cellulose as a polymer material for LB preparation are limited; only one previous study attempted application of nano-porous nitrocellulose LB for wound healing, and presented 
the physicochemical properties and therapeutic effects on cutaneous wound healing in an ICR model (10).

In the current 12-day study, we evaluated the efficacy of on healing cutaneous wounds and toxicity in Sprague Dawley rats after application of LB prepared with cellulose powders from Styela clava tunics (SCT) and Broussonetia kazinoki bark (BSLB). The results of this study provide strong evidence that BSLB is a promising wound dressing due to its acceptable properties for healing wounds and non-toxicity in the injured skin of Sprague Dawley rats.

\section{Materials and methods}

SCT powder was prepared as previously described (20). Briefly, SCT were collected from the beach of the South Sea in Gosung-gun, Korea, and voucher specimens of LP (WPC-14-002) were deposited at the Functional Materials Bank of the PNU-Wellbeing RIS Center in Pusan National University (Miryang, Korea). Sediments and debris were removed by boiling SCT (33 g) in 10\% NaOH (Daejung Co., Gyeonggi-do, Korea) aqueous solution $(990 \mathrm{ml})$ at $100^{\circ} \mathrm{C}$ for $2 \mathrm{~h}$. Samples were subsequently washed three times with distilled water, boiled in $5 \% \mathrm{CH}_{3} \mathrm{COOH}$ (Sigma-Aldrich; Merck KGaA, Darmstadt, Germany) solution at $100^{\circ} \mathrm{C}$ for $2 \mathrm{~h}$ to neutralize the $\mathrm{NaOH}$ solution, and washed thrice in distilled water. SCT was subsequently bleached by boiling and washing separately in $10 \% \mathrm{H}_{2} \mathrm{O}_{2}$ (Junsei Co., Tokyo, Japan) solution. After a final wash with distilled water, the resulting SCT was dried at $100-120^{\circ} \mathrm{C}$ for 2-3 h, followed by grinding in a pin mill machine (Daehwa Co., Goyang, Korea). The milling for SCT powder was conducted by a proprietary commercial process which involved passing through a combination of 30 mesh sieve for 10 min once, and then sieved twice through 120 mesh for 10 min each.

BKB were collected from the cultivation area in Imshil-Gun, Korea, and voucher specimens of LP(WPC-15-001) were deposited at the Functional Materials Bank of the PNU-Wellbeing RIS Center in Pusan National University. Briefly, BKB were heated at $20^{\circ} \mathrm{C}$ for $10 \mathrm{~min}$ and cooled to $1^{\circ} \mathrm{C}$ for $1 \mathrm{~min}$. They were then boiled in $5 \% \mathrm{NaOH}$ and $10 \% \mathrm{Na}_{2} \mathrm{CO}_{3}$ (Junsei Co.) solution $(1,000 \mathrm{ml})$ at $95^{\circ} \mathrm{C}$ for $1 \mathrm{~h}$, followed by washing with tap water for $20 \mathrm{~min}$. This step was repeated with $2 \% \mathrm{NaOH}$ and $3 \% \mathrm{Na}_{2} \mathrm{CO}_{3}$ solution $(1,000 \mathrm{ml})$ using the same conditions. After a final wash with distilled water, the BKB was dried at $100-120^{\circ} \mathrm{C}$ for $2-3 \mathrm{~h}$, before grinding in a pin mill machine (Daehwa Co.).

BSLB was prepared using a modified version of the method described in a previous study (20). To manufacture high concentration BSLB (HiBSLB), $1.5 \mathrm{~g}$ of SCT powder and $1.5 \mathrm{~g}$ of BKB powder were completely dissolved in $100 \mathrm{ml}$ of [Amim] Cl ionic liquid composed of 1-methylimidazole (Daejung Co.) and 3-chloro-1-propene (Sigma-Aldrich; Merck $\mathrm{KGaA} ; 1: 1.20$ of molar ratio) at $80^{\circ} \mathrm{C}$. The low concentration BSLB (LoBSLB) was prepared by diluting HiBSLB $(4.3 \mathrm{ml})$ in $25.17 \mathrm{ml}$ of [Amim] Cl ionic liquid before use (Fig. 1).

Design of animal experiment. The animal protocol used in this study was reviewed and approved by the Pusan National University-Institutional Animal Care and Use Committee (PNU-IACUC; approval no. PNU-2015-0972). Adult male Sprague Dawley rats were purchased from Samtako BioKorea
(Osan, Korea), and handled at the Pusan National University Laboratory Animal Resources Center accredited by the Korea Food and Drug Administration (accredited unit no. 00231) and AAALAC International (accredited unit no. 001525). All rats were provided with a standard irradiated chow diet (Purina Mills, Seoungnam, Korea) ad libitum, and were maintained in a specific pathogen-free (SPF) state under a strict light cycle (lights on at 06:00 h and off at 18:00 h) at a temperature of $23 \pm 2^{\circ} \mathrm{C}$ and a relative humidity of $50 \pm 10 \%$.

An in vivo wound healing assay was developed, in which seven-week-old Sprague Dawley rats $(n=20)$ were assigned to one of four groups: Vehicle (gauze, GZ)-treated group $(n=5)$; Medifoam liquid bandage (MFLB)-treated group $(n=5)$; LoBSLB-treated group $(n=5)$; and HiBSLB-treated group $(n=5)$. MFLB as a positive control bandage was purchased from Mundipharma (Seoul, Korea). Briefly, animals were anesthetized by intramuscular injection with Zoletile $(50 \mathrm{mg} / \mathrm{kg}$ body weight) and Rompun ( $5 \mathrm{mg} / \mathrm{kg}$ body weight). Appropriate anesthesia of all rats was monitored by pedal reflex which clamps the tail or the transitional skin of the rat with forceps and corneal reflex that touches the cornea with watery cotton. After, the back skins were shaved with an electrical razor and cleaned with $70 \%$ ethanol. Using a biopsy punch (Kasco Co., Sialkot, Pakistan), a round wound of $8 \mathrm{~mm}$ diameter and 2-4 mm depth was created by removing the cutaneous tissue in the back shoulder region. The incision wound on each rat was sterilized with $70 \%$ ethanol, after which it was treated with GZ, MFLB, LoBSLB or HiBSLB. The pieces $(5 \times 4 \times 0.3 \mathrm{~mm})$ of GZ and the three types of LB were replaced every 4 days. During replacement, the condition of the skin wound was observed and photographed with a Canon ${ }^{\circledR}$ digital camera, while the body weight was measured using an electronic balance (Mettler Toledo, Greifensee, Switzerland). After 12 days, all rats were euthanized using carbon dioxide $\left(\mathrm{CO}_{2}\right)$ according to the AVMA Guidelines for the Euthanasia of Animals: 2013 Edition (gradual filling at verified $10-30 \%$ displacement rate/min). During this process, $\mathrm{CO}_{2}$ gas was introduced into the 18.741 euthanasia chamber at a flow rate of $3.7 \mathrm{l} / \mathrm{min}$ of $(20 \%$ chamber volume/min). Successful euthanization in rats was confirmed by the measurement of several biological signals including heartbeat, movement, breathing and pupillary response to light. The samples of the damaged skin were collected for further histological and western blot analysis. Additionally, blood serum from the abdominal veins, and liver and kidney tissues were collected to analyze the toxicity of the two BSLBs.

Macroscopic analyses of the surgical wounds. Photographic data were used for measuring the wound size (\%), which was calculated as follows:

$$
\text { Wound size }(\%)=\frac{w t}{w 0}
$$

where Wt is the wound area at time ' $\mathrm{t}$ ' and $\mathrm{W}_{0}$ is the wound area at the initial time. A multiple comparisons test was performed for clarifying the statistical difference between groups.

Serum biochemistry. At day 12, all animals were fasted for $8 \mathrm{~h}$, after which blood was collected from the abdominal veins of rats and incubated for $30 \mathrm{~min}$ at room temperature. Serum 
A Styela clava tunic
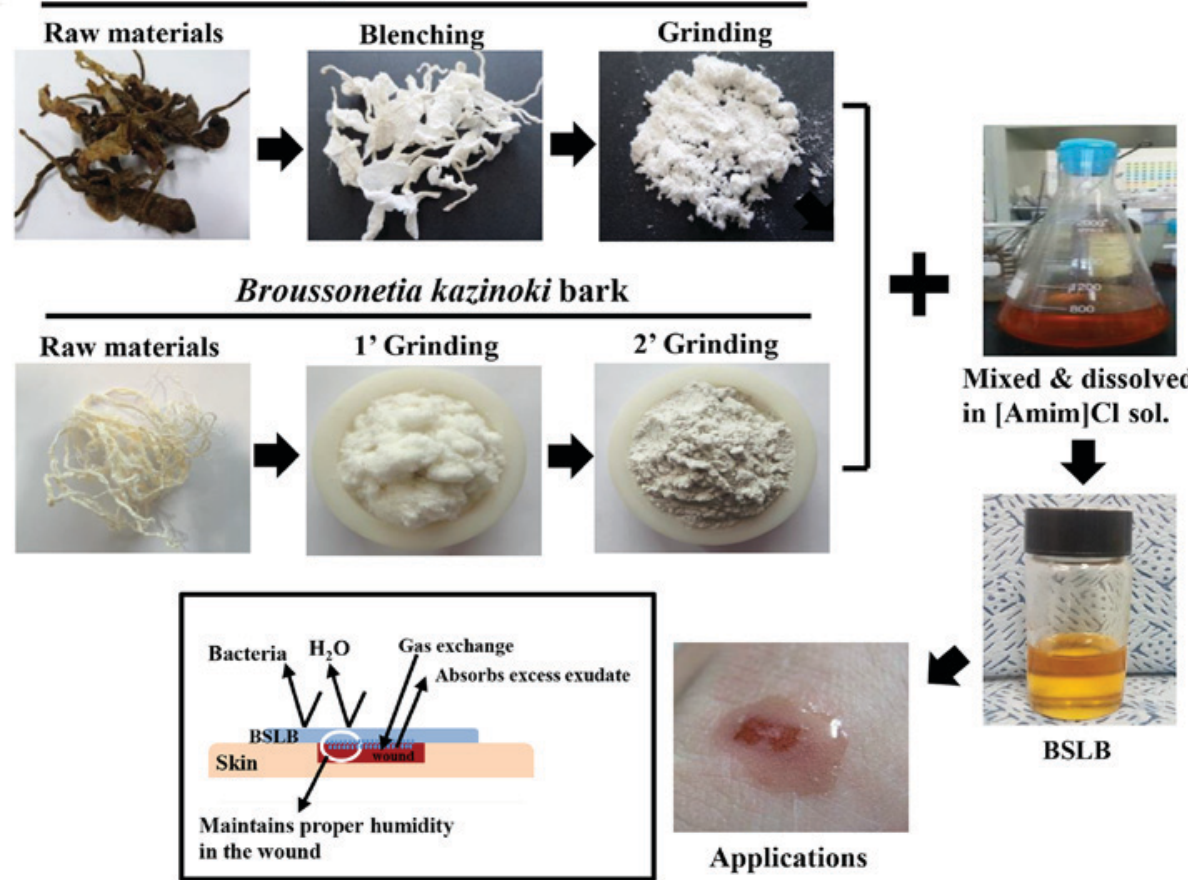

Mixed \& dissolved in [Amim]Cl sol.

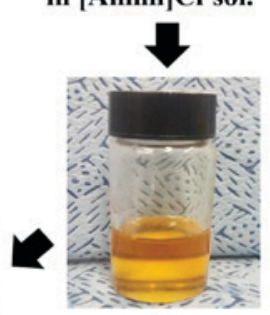

BSLB

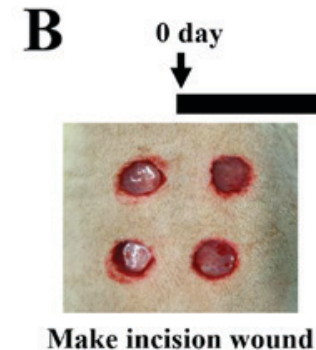

\& LB treatment
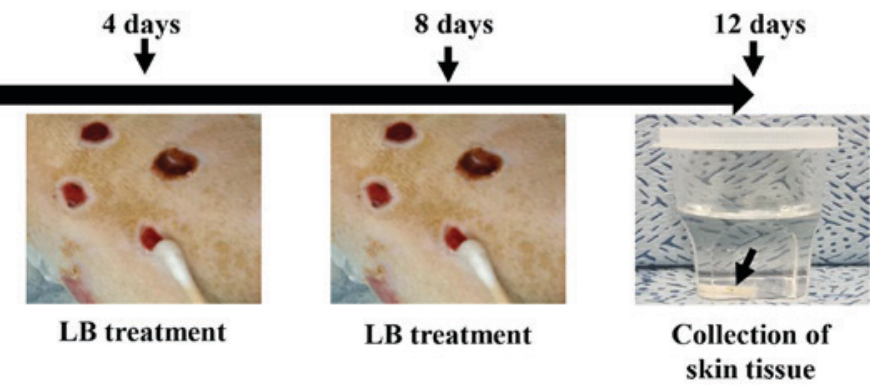

Figure 1. Preparation and treatment of BSLB to the surgical wound injury. (A) BSLB was prepared by serial processing consisting of bleaching, neutralizing, milling, dissolving and mixing. (B) A cutaneous wound measuring $8 \mathrm{~mm}$ in diameter and 2-4 $\mathrm{mm}$ in depth was created by removing the skin tissue using a biopsy punch. These were treated with GZ, MFLB, LoBSLB or HiBSLB, which were replaced every 4 days. BSLB, liquid bandage; GZ, gauze; MFLB, medifoam liquid bandage; LoBSLB, low concentration BSLB; HiBSLB, high concentration BSLB.

was obtained by centrifugation the whole blood, and serum biochemical components, including alkaline phosphatase (ALP), alanine aminotransferase (ALT), aspartate aminotransferase (AST), blood urea nitrogen (BUN), and creatinine (CREA), were assayed using an automatic serum analyzer (Hitachi 747; Hitachi Ltd., Tokyo, Japan). All assays were measured in duplicate using fresh serum.

Histological analyses. The site of application on the cutaneous wound region was collected and fixed in $10 \%$ formalin for $48 \mathrm{~h}$, embedded in paraffin wax, and sectioned into $4 \mu \mathrm{m}$ thick slices. The skin sections were subsequently stained with hematoxylin and eosin (H\&E; Sigma-Aldrich; Merck KGaA) and examined by light microscopy for the presence of edema and inflammatory cell accumulation. Additionally, re-epithelialization and thickness of the epidermis were measured using the Leica Application Suite (Leica Microsystems, Wetzlar, Germany). The liver and kidneys collected from all experimental rats were processed using the same protocol applied to treat the skin tissue, and pathological changes were examined after H\&E staining, using the Leica Application Suite (Leica Microsystems).
Western blot analysis. Granulated wound skin tissue were isolated from a subset of groups, homogenized using a PRO-PREP ${ }^{\mathrm{TM}}$ Solution kit (iNtRON Biotechnology, Sungnam, Korea) supplemented with half tablet of a protein inhibitor cocktail (Roche Applied Science, Penzberg, Germany), and the mixture was centrifuged at $13,000 \mathrm{rpm}$ for $5 \mathrm{~min}$. The prepared proteins were then electrophoresed on a 10\% SDS-PAGE gel, following which they were transferred onto a nitrocellulose membrane (Amersham Biosciences, Corston, UK) for $2 \mathrm{~h}$ at $40 \mathrm{~V}$ in transfer buffer (25 mM Trizma-base, $192 \mathrm{mM}$ glycine, and $20 \%$ methanol). The efficiency of the transfer and equal protein loading were determined by staining the membrane with Ponceau, while the gel was stained with Coomassie blue (both Sigma-Aldrich; Merck KGaA). Appropriate dilutions of the following primary antibodies were added to the membranes and allowed to hybridize overnight at $4^{\circ} \mathrm{C}$ : Anti-VEGF (PeproTech, Rocky Hill, New Jersey, USA), anti-EGFR (Abcam, Cambridge, UK), anti-p-EGFR (Cell Signaling Technology, Inc., Danvers, MA, USA), anti-collagen-1 (Abcam), anti-AKT, anti-phospho-AKT (both Cell Signaling Technology, Inc.), and anti- $\beta$-actin (Sigma-Aldrich; Merck KGaA). After removing 
the antibodies, the membrane was washed three times in a solution composed of $10 \mathrm{mM}$ Trizma-base ( $\mathrm{pH} 7.6), 150 \mathrm{mM} \mathrm{NaCl}$ (both Sigma-Aldrich; Merck KGaA), and 0.05\% Tween-20 (Biosesang, Gyeonggi-do, Korea), for $10 \mathrm{~min}$. The primary antibody conjugated membranes were then incubated with horseradish peroxidase-conjugated anti-secondary antibody for $1 \mathrm{~h}$ at room temperature. The membrane was washed again as described above, and developed using an enhanced chemiluminescence detection system (Amersham Biosciences). Finally, the results were quantified using the Image Analyzer System (Eastman Kodak 2000MM; Kodak, Rochester, NY, USA), and the results are expressed as the fold-increase over control values. All results were confirmed by two independent researchers conducting the experiments at least twice.

Immunohistochemical analysis. Collagen distribution was detected by immunohistochemical analysis using light microscopy, as previously described (21). Briefly, the wound skin tissue samples were fixed in $10 \%$ formalin for $48 \mathrm{~h}$, embedded in paraffin, and sliced into $4 \mu \mathrm{m}$ thick sections. These sections were subsequently deparaffinized with xylene, rehydrated, and pretreated for $30 \mathrm{~min}$ at room temperature with PBS blocking buffer containing $10 \%$ goat serum. The sections were then incubated with primary anti-collagen-1 antibody (Abcam) or anti-p-EGFR (Cell Signaling Technology, Inc.) diluted 1:300 in PBS blocking buffer. The antigen-antibody complexes were subsequently visualized with biotinylated secondary antibody (goat anti rabbit)-conjugated HRP streptavidin (Histostain-Plus kit; Zymed, South San Francisco, CA, USA) at a dilution of 1:1,500 in PBS blocking buffer. Finally, collagen proteins were detected using a stable DAB (Invitrogen; Thermo Fisher Scientific, Inc., Waltham, MA, USA) and the Leica Application Suite (Leica Microsystems). Also, the distribution of p-EGFR protein in skin section was analyzed in the same way using anti-p-EGF receptor antibody.

Statistical analysis. One-way ANOVA (SPSS for Windows, Release 10.10, Standard Version; SPSS Inc., Chicago, IL, USA) followed by Tukey's post hoc test was used to identify significant differences between the Vehicle and the three LB-treated groups. All values are reported as the means $\pm \mathrm{SD}$, and $\mathrm{P}<0.05$ was considered to indicate a statistically significant difference.
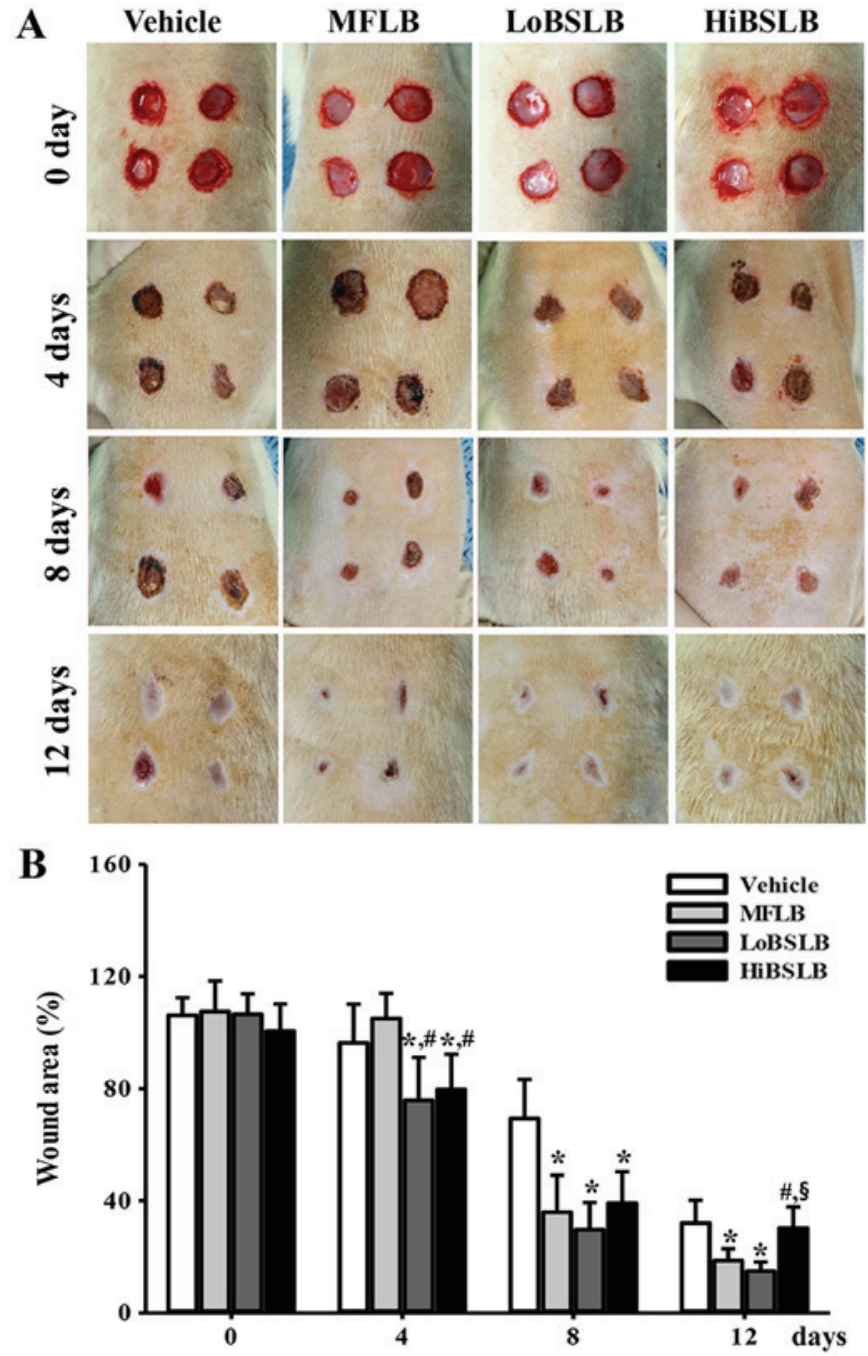

Figure 2. Healing pattern of wounded skin over a time period. At each time point, images of surgical wound skin of rats of each group were taken, and the morphological features were evaluated. (A) Macro-observation of experimental wound healing at various times post-surgery. (B) The ratio of wound area $(\%)$ to initial area on day 1 was measured over time. The wound size was measured in triplicate in each test. Five or six wounds were assayed in triplicate using the wound area analysis. Data are reported as the mean $\pm \mathrm{SD}$. ${ }^{*} \mathrm{P}<0.05$ relative to the Vehicle-treated group; ${ }^{\#} \mathrm{P}<0.05$ relative to the level of the MFLB group, ${ }^{\S} \mathrm{P}<0.05$ relative to the LoBSLB group. BSLB, liquid bandage; MFLB, medifoam liquid bandage; LoBSLB, low concentration BSLB; HiBSLB, high concentration BSLB.

\section{Results}

Effect of BSLB treatments on wound closing process. To determine the stimulatory effects of BSLB on the healing process of wounded skin, a spherical area measuring $8 \mathrm{~mm}$ in diameter and 2-4 mm in depth was evaluated for wounded skin covered with LoBSLB and HiBSLB for 12 days. The most significant changes observed in the wound area occurred between days 4-12. In the LoBSLB-treated group, the wound area decreased $79 \%$ on day $4,43 \%$ on day 8 , and $47 \%$ on day 12 , when compared to the Vehicle-treated group. Similar results were also observed in the HiBSLB-treated group, with complete disappearance of the wound on day 12 (Fig. 2A and B). Furthermore, the decrease in wound area in the MFLB-treated group was similar to the LoBSLB-treated group (Fig. 2A and B). These results suggest that enhanced healing ability of the cutaneous surgical wound is reasonably attributed to BSLB treatment.
Effect of BSLB treatment on tissue regeneration of wounded skin. The alterations in tissue regeneration of the wounded skin was determined by the recovery of the epidermis, dermis and hyperdermis of the skin tissue of rats over the designated study duration (12 days). To measure the therapeutic effects of the BSLB on tissue regeneration in the surgical skin wounds, we measured the thickness of the epidermis and the width of re-epithelialization region in the Vehicle, MFLB, LoBSLB and HiBSLB-treated groups. We observed a significant increase in re-epithelialization in the LoBSLB and HiBSLB-treated groups relative to the Vehicle-treated group, with a greater increase rate in the LoBSLB group than the HiBSLB group (Fig. 3A). Additionally, a similar pattern was observed for the thickness of the total epidermis. After day 12 post-surgery, the thickness of the total epidermis was significantly enhanced by about 
A

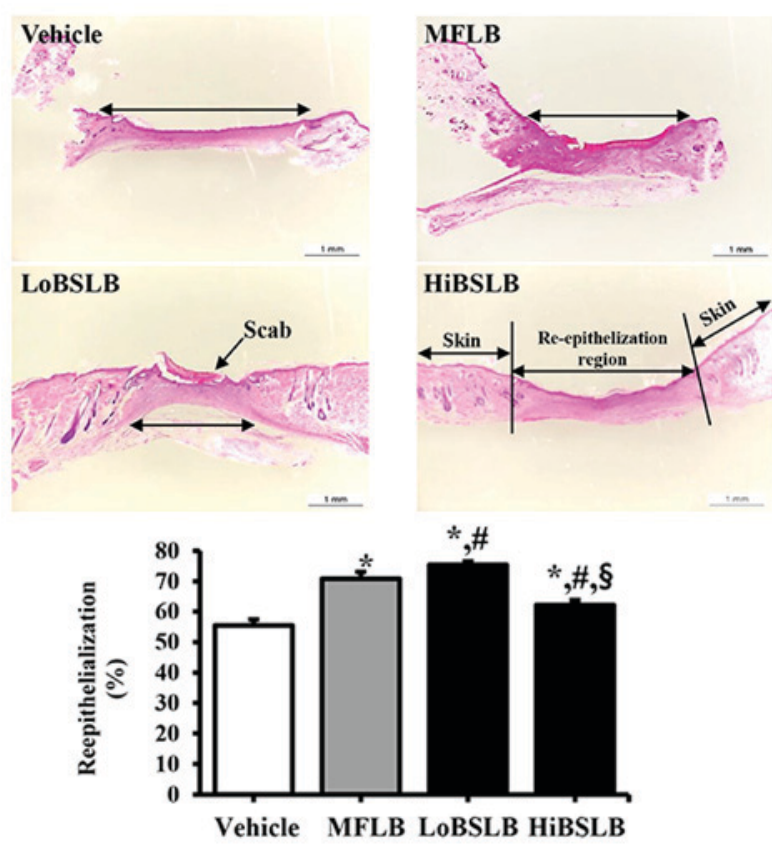

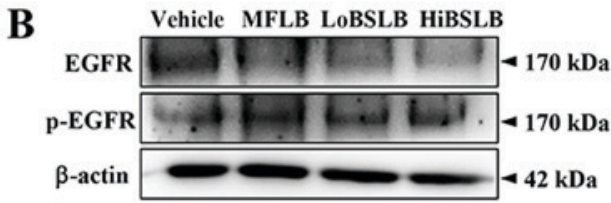

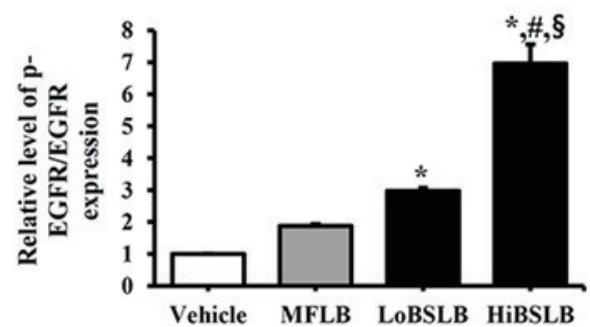

C
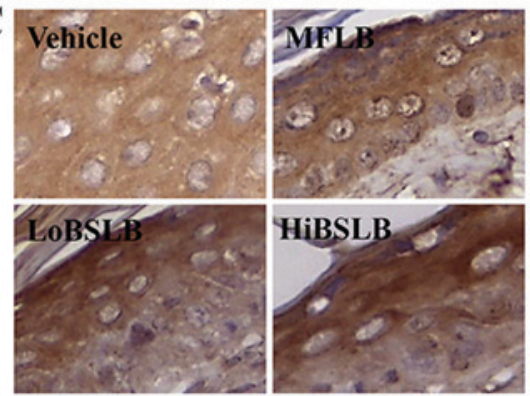

Figure 3. Re-epithelialization analysis after BSLB application. (A) H\&E stained sections of subcutaneous tissue surrounding the applications of BSLB at day 12 were observed using a light microscope at a magnification, x100. Five or six wounds were assayed in triplicate using wound area analysis. (B) Analysis of EGFR phosphorylation. Western blot analysis of EGFR, p-EGFR and $\beta$-actin in a homogenate mixture prepared from the wound skin was detected using the specific primary antibodies. (C) The distribution of p-EGFR were also observed in skin tissue at 400x magnification, after staining with p-EGFR antibody. Values are defined as 1 (Vehicle-treated group) for the acquisition of other relative values. Data are reported as the mean \pm SD. "P<0.05 relative to the Vehicle-treated group; ${ }^{*} \mathrm{P}<0.05$ relative to the level of the MFLB group, ${ }^{\circ} \mathrm{P}<0.05$ relative to the LoBSLB group. BSLB, liquid bandage; MFLB, medifoam liquid bandage; LoBSLB, low concentration BSLB; HiBSLB, high concentration BSLB; H\&E, hematoxylin and eosin.

126 and $139 \%$ in the LoBSLB and MFLB-treated groups, respecrively, relative to the Vehicle-treated group; however, constant levels were maintained in the HiBSLB-treated group (Fig. 4A and B). To further determine if the therapeutic effects of BSLB were related with activation of EGFR, we examined for differences in the level of phosphorylation of EGFR in the wound skin of BSLB-treated groups. We observed a significant increase in levels in the HiBSLB-treated group, whereas other groups maintained a constant level (Fig. 3B). Also, a similar pattern was observed in the immunohistochemical analysis for p-EGFR (Fig. 3C). Taken together, these results suggest that BSLB treatment improves tissue regeneration and re-epithelialization in the skin tissue of Sprague Dawley rats through the stimulation of EGFR.

Effect of BSLB on the angiogenesis signaling pathway in wounded skin. The effect of BSLB treatment on the angiogenesis signaling pathway in the dermis of wounded skin was evaluated by measuring the expression level of VEGF and a member of the downstream signaling pathway, in a subset of groups at day 12. On day 12, the expression of VEGF, an important signaling protein involved in both vasculogenesis and angiogenesis, dramatically decreased in the LoBSLB and HiBSLB-treated groups, as compared with Vehicle-treated group (Fig. 5). A similar decrease was observed in AKT phosphorylation, one of members of the VEGF downstream signaling pathway. The phosphorylation of AKT was lower in the LoBSLB and HiBSLB-treated groups, as compared to the Vehicle-treated group, on day 12. These effects were similarly observed in the MFLB-treated group (Fig. 5). These results therefore indicate that BSLB treatment induces the down-regulation of the angiogenesis signaling pathway during the late stage of the wound skin repair process by regulating the expression of VEGF and AKT phosphorylation. 

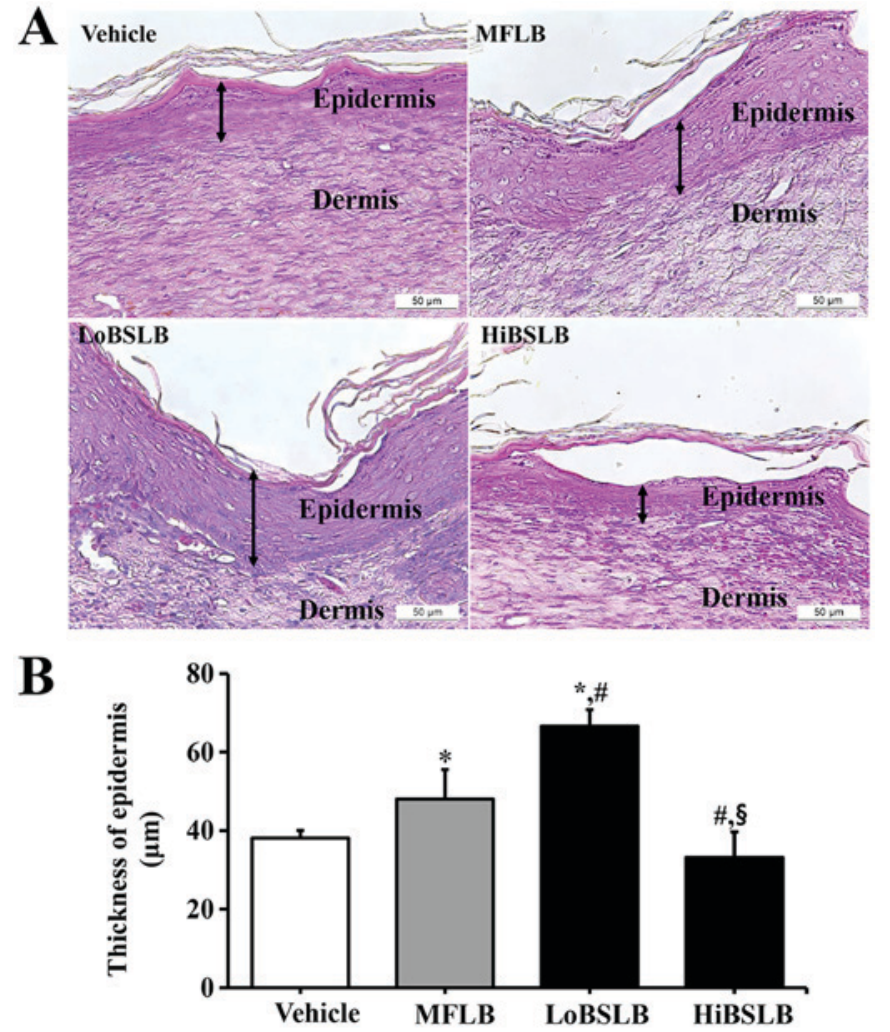

Figure 4. Analysis of epidermal thickness after BSLB application. (A) H\&E stained sections of subcutaneous tissue surrounding the two BSLB applications to Sprague Dawley rats at day 12 were observed using a light microscope at magnification, $\mathrm{x} 400$. (B) Thickness was measured using the Leica Application Suite. Five or six wounds were assayed in triplicate using wound area analysis. Data are reported as the mean $\pm \mathrm{SD}$. ${ }^{*} \mathrm{P}<0.05$ relative to the Vehicle-treated group; ${ }^{\text {"P }}<0.05$ relative to the level of the MFLB group, ${ }^{\S} \mathrm{P}<0.05$ relative to the LoBSLB group. BSLB, liquid bandage; MFLB medifoam liquid bandage; LoBSLB, low concentration BSLB; HiBSLB, high concentration BSLB; H\&E, hematoxylin and eosin.

Effect of BSLB on the formation of connective tissue in wound skin. To evaluate the therapeutic effects of BSLB on the formation of connective tissue in the wounded skin, the expression level of collagen was measured in a subset of groups at different times. We observed a rapid increase in the expression of collagen-1 in the LoBSLB and HiBSLB-treated groups (169 and $137 \%$, respectively) compared with the Vehicle-treated group (Fig. 6A). The wound skin tissue stained for immunohistochemical analysis also showed a similar trend. The collagen protein was densely stained around the dermis of the LoBSLB and HiBSLB-treated groups relative to the Vehicle-treated group (Fig. 6B). These findings indicate that BSLB treatment induces an enhancement in collagen during the late stage of the wounded skin repair process.

Toxicity of BSLB. The toxicity of BSLB toward the liver and kidneys of Sprague Dawley rats was evaluated by investigating modifications in the metabolic enzymes (in the blood serum) and histopathology (of liver and kidney tissues), using serum biochemical analysis and histological analysis, respectively. Liver toxicity analysis revealed no increase in the levels of the three liver toxicity indicators, specifically ALP, AST and ALT, in both the LoBSLB and HiBSLB-treated groups and Vehicle-treated group. Also, no significant pathological
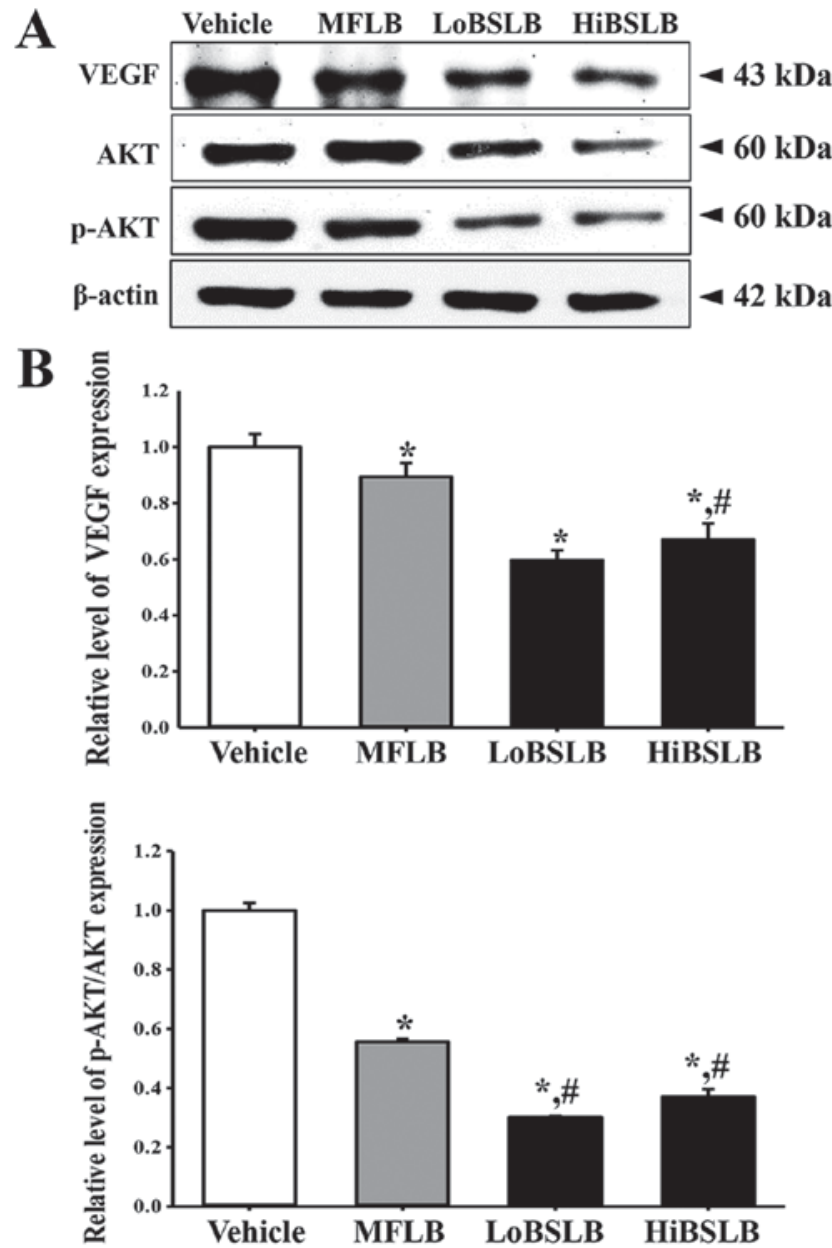

Figure 5. Analysis of the downstream signaling pathway of VEGF for angiogenesis. (A) Western blot analysis of VEGF, AKT, p-AKT and $\beta$-actin was assessed in the homogenate mixture prepared from the wound skin using the specific primary antibodies. (B) Value was defined as 1 (Vehicle-treated group) for the acquisition of other relative values. Data represents the means \pm SD from three replicates. ${ }^{*} \mathrm{P}<0.05$ relative to the Vehicle-treated group; ${ }^{\#} \mathrm{P}<0.05$ relative to the level of the MFLB group, ${ }^{\S} \mathrm{P}<0.05$ relative to the LoBSLB group. BSLB, liquid bandage; MFLB, medifoam liquid bandage; LoBSLB, low concentration BSLB; HiBSLB, high concentration BSLB.

changes such as inflammation, necrosis, apoptosis, or fibrosis were observed in the liver tissue of BSLB-treated groups (Fig. 7). Results for kidney toxicity were similar to the liver toxicity results. The BUN and CREA levels in the serum showed no significant increase in the two BSLB-treated groups, regardless of the dose administered. Furthermore, no specific pathological symptoms, including degeneration and necrosis of the glomerulus and renal tubes, were detected in the kidney tissue of the BSLB-treated groups (Fig. 8). The above results provide strong evidence that treatment with BSLB for a short duration does not induce any significant toxicity in the liver and kidneys of Sprague Dawley rats.

\section{Discussion}

Various medical dressings have been extensively applied for treating external cutaneous wounds such as abrasions, lacerations, avulsions, puncture wounds, contusions, blisters, incisions, burns, split graft donor sites and ulcers (22). LB has recently received a great deal of attention as a novel method of 


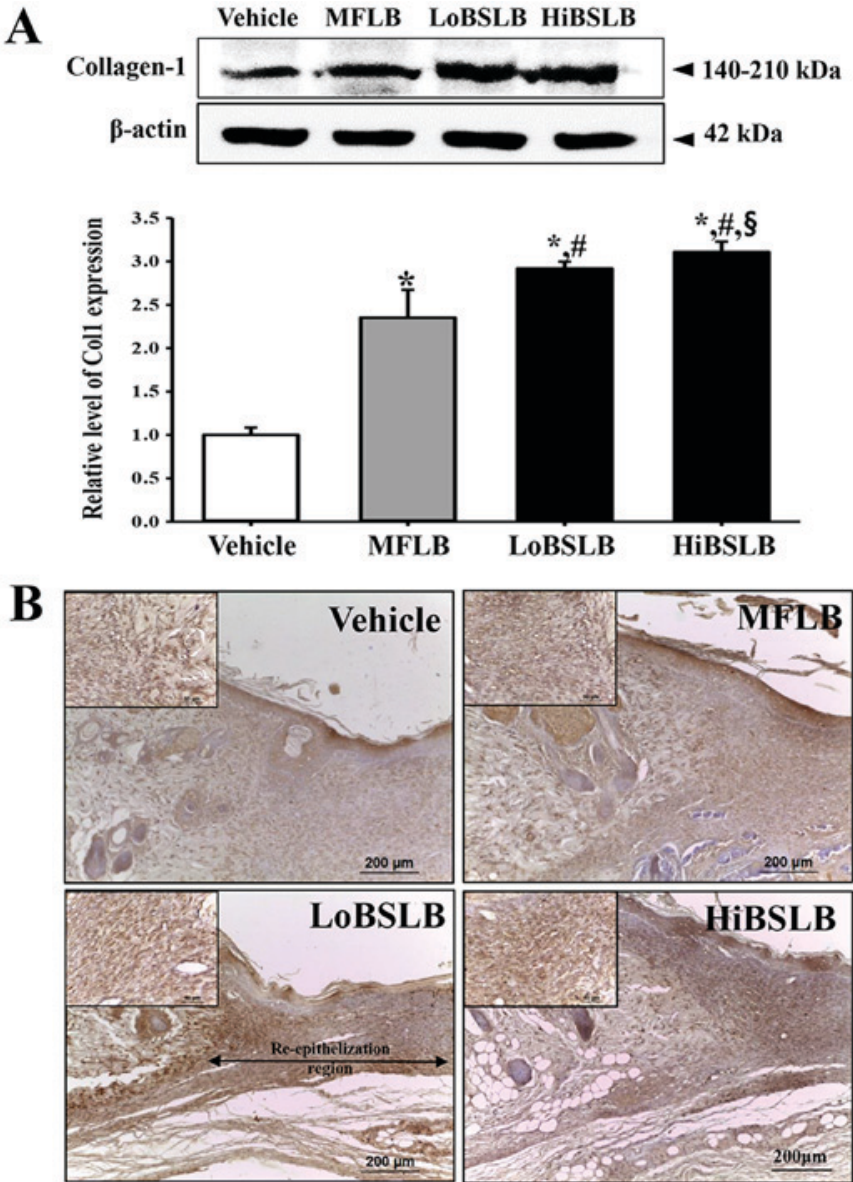

Figure 6. Analysis of collagen-1 expression. (A) Western blot analysis of collagen-1 in the homogenate mixture prepared from the wound skin was evaluated using the specific primary antibody. The value was defined as 1 (Vehicle-treated group) for the acquisition of other relative values. Data represents the mean $\pm \mathrm{SD}$ of three replicates. ${ }^{*} \mathrm{P}<0.05$ relative to the Vehicle-treated group; ${ }^{*} \mathrm{P}<0.05$ relative to the level of the MFLB group; ${ }^{\S} \mathrm{P}<0.05$ relative to the LoBSLB group. (B) Immunohistochemistry of collagen protein. After BSLB treatment, the collagen-1 proteins were detected as a dense color around the dermis of re-epithelization region, when compared with the Vehicle-treated group. Histological alteration was observed around the re-epithelization region stained with specific antibody at magnification, x100 and x400 (left upper corner). BSLB, liquid bandage; MFLB, medifoam liquid bandage; LoBSLB, low concentration BSLB; HiBSLB, high concentration BSLB.

medical dressings for the treatment of various wounds, since it involves decreased trauma and eliminates the use of sutures and staples (13). This study therefore attempted to obtain novel evidence for the potential medical applications of BSLB, and further investigated the therapeutic effects and toxicity in surgical cutaneous wounds of Sprague Dawley rats. Our results clearly indicate that BSLB accelerates the recovery of wounded skin, re-epithelialization, granular tissue formation, and angiogenesis, without any accompanying toxicity.

Our results demonstrate that BSLB treatment for 12 days stimulated wound closure in surgically injured skin of Sprague Dawley rats. Decrease in the wound area, as well as enhancement of re-epithelialization was strongly detected within 12 days of surgical injury, as presented in Figs. 2 and 3. These results were consistent with a previous study using topical application of nano-porous nitrocellulose LB. The cellulose significantly increased the wound closure as compared to the control group in ICR mice, although their change rate varied
A
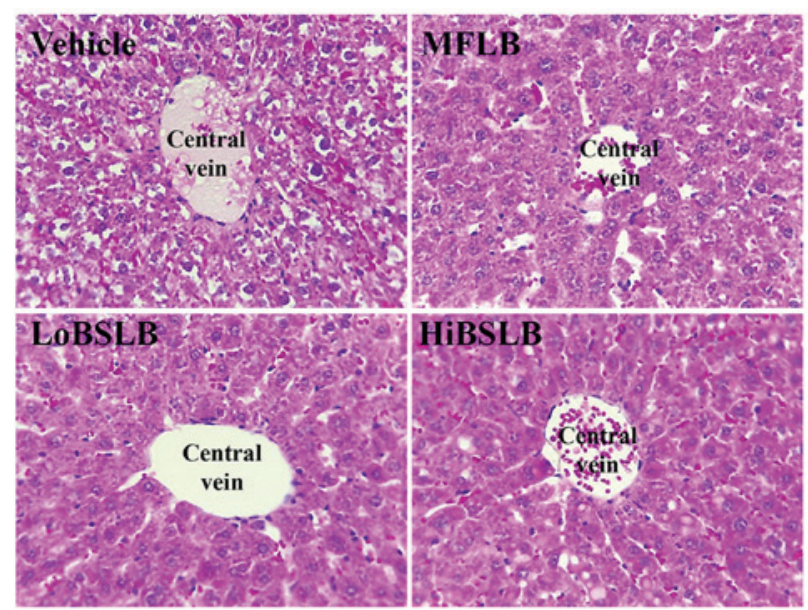

B
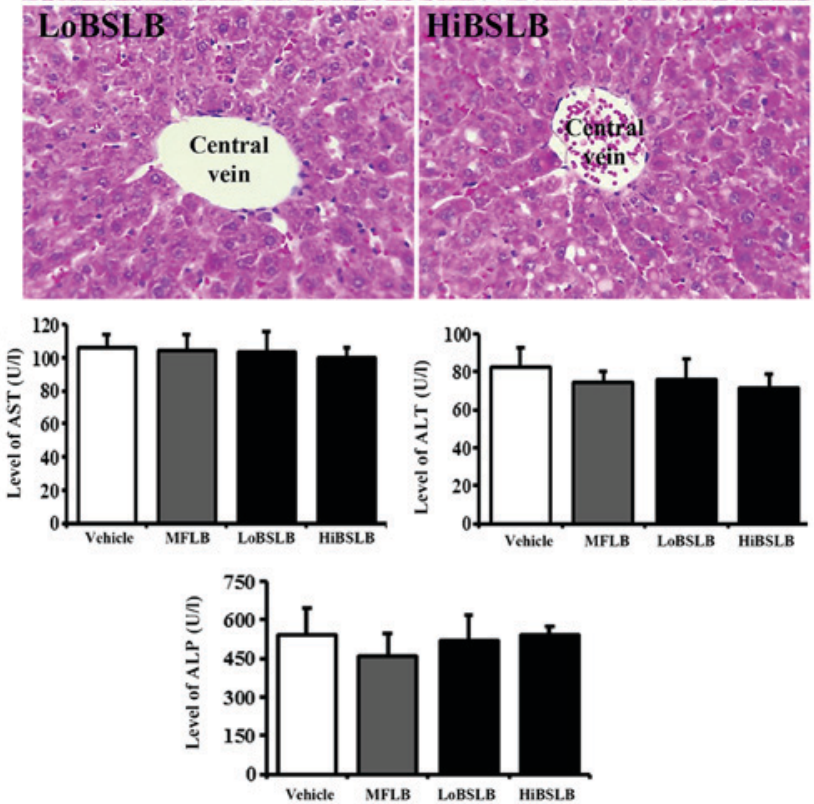

7. Evaluation of hepatotoxicity of BSLB application. (A) Liver tissue of Sprague Dawley rats was prepared on a histological slide, and cellular morphology was viewed at magnification, $\mathrm{x} 400$. (B) Blood was collected from the abdominal veins of Vehicle and the two BSLB-treated Sprague Dawley rats. Serum concentrations of ALP, AST and ALT were analyzed in duplicate using a serum biochemical analyzer. Data represents the mean \pm SD from three replicates. BSLB, liquid bandage; MFLB, medifoam liquid bandage; LoBSLB, low concentration BSLB; HiBSLB, high concentration BSLB; ALP, alkaline phosphatase; ALT, alanine aminotransferase; AST, aspartate aminotransferase.

in each group (10). These two studies therefore show that LB prepared with cellulose powder promotes the rate of cutaneous wound healing.

The current study further concludes that BSLB treatment accelerates the regeneration of surgical wound in the skin tissue of Sprague Dawley rats. As presented in Figs. 3 and 4, the re-epithelialization rate and thickness of epidermis is significantly enhanced in the LoBSLB-treated group compared with Vehicle-treated group. Similar results were obtained for surgical wounds in ICR mice-treated with nano-porous nitrocellulose LB, where application of the nitrocellulose LB onto surgical skin wounds exhibited enhanced wound re-epithelialization, contraction and granular tissue formation when compared with control-treated group (10). However, the results obtained in the HiBSLB-treated group was dissimilar; we observed a constant level of epidermal thickening and only slight increase of re-epithelialization rate at day 12 . Hence, we believe that LoBSLB treatment is more suitable for wound regeneration than HiBSLB treatment. The lower therapeutic effect of HiBSLB in wound regeneration could be a consequence of varying concentrations of components containing cellulose, even though cellulose was the basic building material. 

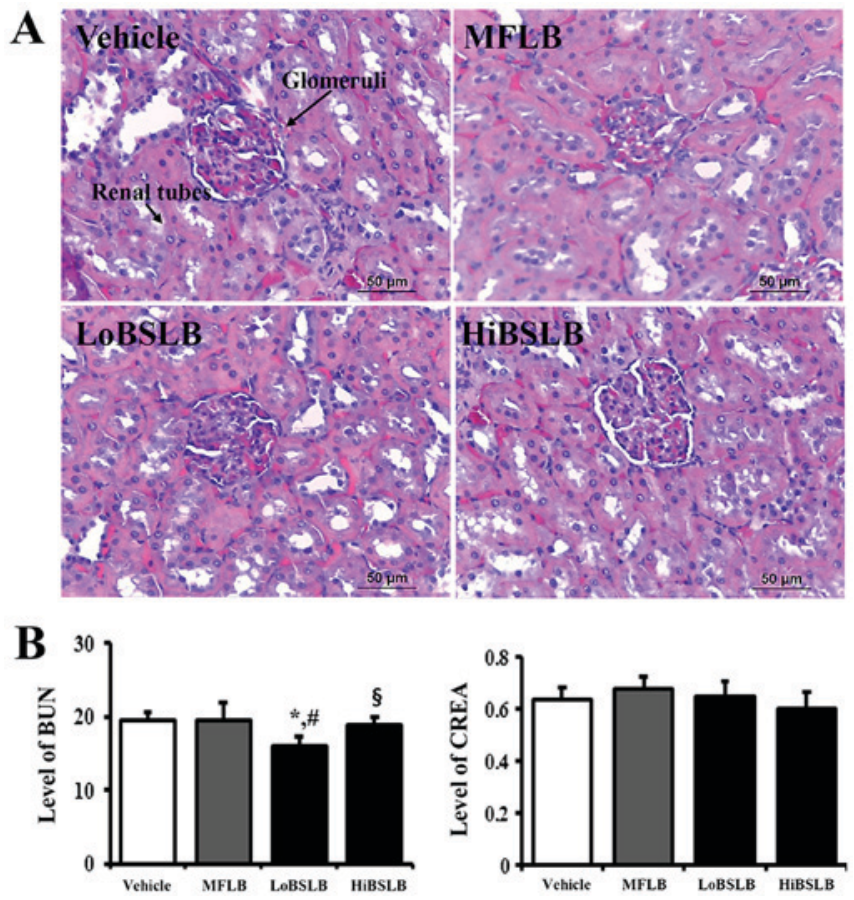

Figure 8. Evaluation of nephrotoxicity of BSLB application. (A) Kidney tissue of Sprague Dawley rats was prepared on a histological slide, and cellular morphology was viewed at magnification, $x 400$. (B) Blood was collected from the abdominal veins of Vehicle and the two BSLB-treated Sprague Dawley rats. Serum concentrations of BUN and CREA were analyzed in duplicate, using a serum biochemical analyzer as described in the Materials and Methods. Data represents the mean $\pm \mathrm{SD}$ from three replicates. ${ }^{*} \mathrm{P}<0.05$ relative to the Vehicle-treated group; ${ }^{\#} \mathrm{P}<0.05$ relative to the level of the MFLB group; ${ }^{\circledR} \mathrm{P}<0.05$ relative to the LoBSLB group. BSLB, liquid bandage; MFLB, medifoam liquid bandage; LoBSLB, low concentration BSLB; HiBSLB, high concentration BSLB; BUN, blood urea nitrogen; CREA, creatinine.

Angiogenesis is an integral component of normal wound repair since it provides the nutrients required by the healing tissue and contributes to the structural repair by stimulating the formation of granulation tissue (23). During angiogenesis, VEGF enhances the permeability, growth and migration of endothelial cells on the surface of a collagen matrix (24-26). This activity is also partially mediated via the MAPK2K1/2/MAPK3/1 or PI3K/AKT1 pathways (27-29). The expression of VEGF gradually elevates after full a thickness skin wound, and reaches maximal level at approximately days 3 to 7 during the period of granulation tissue formation. Once it reaches the remodeling phase, the VEGF signaling pathway gradually disappears (30). Therefore, evaluating the decrease in the expression of key component of the VEGF signaling pathway can be considered a key marker indicating the effects of tissue repair. The decrease of VEGF expression has been widely examined after several therapeutic treatments for wound repair, although there are variations in the duration of observation. Topical application of Substance S significantly increased the wound closure and decreased VEGF expression at day 14 in rats with open excision wound (31). The VEGF mRNA expression was down-regulated in all scars-treated with Ginsenoside-Rg3 ( $\operatorname{Rg} 3$ ) compared to the control group (32). Similarly, our study revealed decreased VEGF expression in the wound skin of LoBSLB and HiBSLB-treated groups.

Meanwhile, collagen is an important structural protein of the extracellular matrix and has a key role in all phases of surgical wound healing (33). An improved deposition of collagen was reported in surgical wounded skin-treated with nano-porous nitrocellulose LB after Sirius Red staining (10). In the present study, the expression of collagen increased in a dose dependent manner in the LoBSLB and HiBSLB-treated groups, as compared with the Vehicle-treated group, on day 12. These results are similar to previous studies, although the analysis tool was differed in each study.

The toxicity of LB prepared with cellulose powders has so far not been investigated in mammalian systems. However, toxicity for membrane forms prepared by cellulose mixtures are reported by several studies. Specific toxicity with respect to body weight or metabolic enzymes of the liver and kidney was not induced in the Sprague Dawley rats-treated with hydrocolloid membrane (HCM)-SCT for 11 days (34). Also, the concentration of metabolic enzymes representing liver and kidney toxicity was maintained at a constant level in the serum of SCT-cellulose film (CF) implanted Sprague Dawley rats, relative to the vehicle implanted group (20). Similar results for toxicity were also observed in our study using SCT and BKB. Most indicators of liver and kidney toxicity were maintained at a normal level in the LoBSLB and HiBSLB-treated groups throughout the experimental period. These results suggest that natural source cellulose can extensively be applied to surgical injuries without occurrence of any significant toxicity.

Taken together, the results of the present study indicate that topical application of two different concentrations of BSLBs for 12 days induces accelerated surgical wound healing, including tissue regeneration and connective tissue formation. The LoBSLB showed greater therapeutic effect of surgical wound healing of the back skin of Sprague Dawley rats. No significant toxicity was observed in the liver and kidneys of the experimental animals. Overall, these results serve as a rationale for future development of BSLB with other functional compounds, for topical application to cutaneous wounds.

\section{Acknowledgments}

The authors would like to thank Miss Jin Hyang Hwang, the animal technician, for directing the animal care at the Laboratory Animal Resources Center in Pusan National University.

\section{Funding}

This research was supported by grants to Dr. Dae Youn Hwang from the Korea Institute of Planning Evaluation for Technology of Food, Agriculture, Forestry and Fisheries (116027-032-HD030), and a National Research Foundation of Korea (NRF) grant funded by the Korean government (MSIP) (grant no. MRC, 2008-0062275).

\section{Availability of data and materials}

The datasets used and/or analyzed during the current study are available from the corresponding author on reasonable request.

\section{Authors' contributions}

JJP, JEK, WBY, MRL, JYC, BRS and DYH participated in the design of the study, sample preparation, animal experiments 
and data analyses. H-JS, YL, H-GK, BSA, SYY and SBS assisted with data analysis and manuscript preparation. All authors read and approved the final manuscript.

\section{Ethics approval and consent to participate}

The present study complied with the ethics standard for research activity established at Pusan National University. The animal protocol for this study was reviewed and approved based on the ethical procedures for scientific care by the Pusan National University-Institutional Animal Care and Use Committee (PNU-IACUC; approval no. PNU-2015-0972).

\section{Patient consent for publication}

Not applicable.

\section{Competing interests}

The authors declare that they have no competing interests.

\section{References}

1. Crépy L, Monchau F, Chai F, Raoul G, Hivart P, Hildebrand HF Martin P and Joly N: Evaluation of a bio-based hydrophobic cellulose laurate film as biomaterial-study on biodegradation and cytocompatibility. J Biomed Mater Res B Appl Biomater 100 1000-1008, 2012.

2. Lavoine N, Desloges I, Dufresne A and Bras J: Microfibrillated cellulose-its barrier properties and applications in cellulosic materials: A review. Carbohydr Polym 90: 735-764, 2012.

3. Spencer PC, Schmidt B, Samtleben W, Bosch T and Gurland HJ: Ex vivo model of hemodialysis membrane biocompatibility. Trans Am Soc Artif Intern Organs 31: 495-498, 1985.

4. Gissinger D and Stamm A: A comparative study of cross-linked carboxymethylcellulose as tablet disintegrant. Pharm Ind 42 : 189-192, 1980

5. Franz G: Polysaccharides in pharmacy. Adv Polymer Sci 76 $1-30,1986$.

6. Ito $\mathrm{H}$, Shibata T, Miyamoto T, Noishiki $\mathrm{Y}$ and Inagaki $\mathrm{H}$ : Formation of polyelectrolyte complexes between cellulose derivatives and their blood compatibility. J Appl Polym Sci 31: 2491-2500, 1986.

7. Matasuzaki K, Yamamoto I, Sato T and Oshima R: Synthesis of water-soluble branched polysaccharides and their antitumor activity, 1 . Branched polysaccharides from cellulose acetate. Macromol Chem Phys 186: 449-456, 1985.

8. Lee CG, Da Silva CA, Lee JY, Hartl D and Elias JA: Chitin regulation of immune responses: An old molecule with new roles. Curr Opin Immunol 20: 684-689, 2008.

9. Hirano S: Chitin biotechnology applications. Biotechnol Annu Rev 2: 237-258, 1996

10. Mu X, Yu H, Zhang C, Chen X, Cheng Z, Bai R, Wu X, Yu Q, $\mathrm{Wu} \mathrm{C}$ and Diao Y: Nano-porous nitrocellulose liquid bandage modulates cell and cytokine response and accelerates cutaneous wound healing in a mouse model. Carbohydr Polym 136: 618-629, 2016.

11. Fricain JC, Granja PL, Barbosa MA, de Jéso B, Barthe N and Baquey C: Cellulose phosphates as biomaterials. In vivo biocompatibility studies. Biomaterials 23: 971-980, 2002.

12. Martson M, Viljanto J, Hurme T, Laippala P and Saukko P: Is cellulose sponge degradable or stable as implantation material? An in vivo subcutaneous study in the rat. Biomaterials 20 : 1989-1995, 1999

13. Petkewich R: Liquid bandages. Chem Eng News 86: 61, 2008.

14. Choi SJ, Lee JH, Lee YH, Hwang DY and Kim HD: Synthesis and properties of polyurethane-urea-based liquid bandage materials. J Appl Polym Sci 121: 3516-3524, 2011.
15. Shepler TR and Seiff SR: Use of isobutyl cyanoacrylate tissue adhesive to stabilize external eyelid weights in temporary treatment of facial palsies. Ophthalmic Plast Reconstr Surg 17: 169-173, 2001.

16. Taravella MJ and Chang CD: 2-Octyl cyanoacrylate medical adhesive in treatment of a corneal perforation. Cornea 20 : 220-221, 2001.

17. Suhr MA, Günther M and Springer IN: Ptosis relief after botox injection using dermabond. Plast Reconstr Surg 114: 262-263, 2004

18. Osaki TH, Osaki MH, Belfort R Jr, Osaki T, Sant'anna AE and Haraguchi DK: Management of progressive myopathic blepharoptosis with daily application of octyl-2-cyanoacrylate liquid bandage. Ophthalmic Plast Reconstr Surg 25: 264-266, 2009.

19. Osaki TH, Osaki MH and Osaki TH: Temporary management of involutional entropion with octyl-2-cyanoacrylate liquid bandage application. Arq Bras Oftalmol 73: 120-124, 2010.

20. Song SH, Kim JE, Lee YJ, Kwak MH, Sung GY, Kwon SH, Son HJ, Lee HS, Jung YJ and Hwang DY: Cellulose film regenerated from Styela clava tunics have biodegradability, toxicity and biocompatibility in the skin of SD rats. J Mater Sci Mater Med 25: 1519-1530, 2014.

21. Hwang DY, Chae KR, Kang TS, Hwang JH, Lim CH, Kang HK, Goo JS, Lee MR, Lim HJ, Min SH, et al: Alterations in behavior, amyloid beta-42, caspase-3, and Cox-2 in mutant PS2 transgenic mouse model of Alzheimer's disease. FASEB J 16: 805-813, 2002.

22. Eaglstein WH: Moist wound healing with occlusive dressings: A clinical focus. Dermatol Surg 27: 175-181, 2001.

23. Nissen NN and DiPietro LA: Angiogenic mediators in healing wounds. In: angiogenesis in Health and Disease. Gabor MR (ed.). Marcel Dekker Inc., New York, 417-427, 2000.

24. Ferrara N: Vascular endothelial growth factor and the regulation of angiogenesis. Recent Prog Horm Res 55: 15-36, 2000.

25. Ferrara N: Role of vascular endothelial growth factor in regulation of physiological angiogenesis. Am J Physiol Cell Physiol 208: C1358-C1366, 2001.

26. Taub PJ, Silver L and Weinberg H: Plastic surgical perspectives on vascular endothelial growth factor as gene therapy for angiogenesis. Plast Reconstr Surg 105: 1034-1042, 2000.

27. Gerber HP, McMurtrey A, Kowalski J, Yan M, Keyt BA, Dixit V and Ferrara N: Vascular endothelial growth factor regulates endothelial cell survival through the phosphatidylinositol 3'-kinase/Akt signal transduction pathway. Requirement for Flk-1/KDR activation. J Biol Chem 273: 30336-30343, 1998.

28. Abid MR, Guo S, Minami T, Spokes KC, Ueki K, Skurk C, Walsh K and Aird WC: Vascular endothelial growth factor activates PI3K/Akt/forkhead signaling in endothelial cells. Arterioscler Thromb Vasc Biol 24: 294-300, 2004.

29. Zheng J, Wen Y, Song Y, Wang K, Chen DB and Magness RR: Activation of multiple signaling pathways is critical for fibroblast grow th factor 2- and vascular endothelial growth factor-stimulated ovine fetoplacental endothelial cell proliferation. Biol Reprod 78: $143-150,2008$.

30. Frank S, Hübner G, Breier G, Longaker MT, Greenhalgh DG and Werner S: Regulation of vascular endothelial growth factor expression in cultured keratinocytes. Implications for normal and impaired wound healing. J Biol Chem 270: 12607-12613, 1995.

31. Kant V, Gopal A, Kumar D, Bag S, Kurade NP, Kumar A, Tandan SK and Kumar D: Topically applied substance P enhanced healing of open excision wound in rats. Eur J Pharmacol 715: 345-353, 2013.

32. Sun X, Cheng L, Zhu W, Hu C, Jin R, Sun B, Shi Y, Zhang Y and Cui W: Use of ginsenoside Rg3-loaded electrospun PLGA fibrous membranes as wound cover induces healing and inhibits hypertrophic scar formation of the skin. Colloids Surf B Biointerfaces 115: 61-70, 2014.

33. Mian M, Beghè F and Mian E: Collagen as a pharmacological approach in wound healing. Int J Tissue React 14 (Suppl): S1-S9, 1992.

34. Kwak MH, Go J, Kim JE, Lee YJ, Lee SH, Lee HS, Son HJ, Jung YJ and Hwang DY: Property and efficacy analysis of hydrocolloid membrane containing Styela clava tunic on the wound repair of skin in SD rats. Biomat Res 17: 91-101, 2013.

This work is licensed under a Creative Commons Attribution-NonCommercial-NoDerivatives 4.0 International (CC BY-NC-ND 4.0) License. 